\title{
DARK MATTER IN THE MILKY WAY
}

\author{
KONRAD KUIJKEN \\ Kapteyn Astronomical Instituut \\ P.O. Box 800, 9700 AV, Groningen, the Netherlands \\ and \\ Harvard-Smithsonian Center for Astrophysics \\ 60 Garden Street, Cambridge MA 02138, USA \\ (kuijken@astro.rug.nl)
}

\section{Introduction}

If the Newtonian inverse-square law is an appropriate description of gravity, then disk galaxies, and the Milky Way in particular, contain large amounts of dark matter: in general, the observed shapes of rotation curves do not correspond to the gravitational potential of the observed stars and gas. The nature of this dark matter is still a topic of debate, the outcome of which will have important consequences for our ideas of how galaxies and larger structures in the universe formed.

The study of the distribution of dark matter in the Milky Way is a venerable and extensive subject; important aspects of it are well-reviewed by Fich and Tremaine (1991), Merrifield (1993) and Schechter (1993).

This review will concentrate on two related topics which are particularly relevant to stellar populations: (1) how stellar populations can be used as tracers of the gravitational potential, and thereby reveal the dark matter distribution within the Galaxy, and (2) the question whether the dark matter is a stellar population.

\section{Stellar Populations as Tracers of the Gravitational Potential}

Stars move under the influence of gravity, so the study of their motions offers a constraint on the strength of the gravitational field, and hence on the overall mass density causing it. Reliable results can only be obtained by studying a stellar population in equilibrium: local features such as Gould's belt in the distribution of $\mathrm{O}$ stars, for instance, say much more about how 
this group of stars was formed than about the Galactic potential (Comeron et al., 1992). Since stars are born in clusters or associations, it is important that they be given sufficient time to spread out before they constitute a dynamically relaxed (which for these purposes means phase-mixed) population.

For geometrical reasons, studies have concentrated on the gravitational force in two directions: in the galactic plane (i.e., the rotation curve) and orthogonal to the plane from the solar neighbourhood (the "solar cylinder"). In both of these cases, the effects of motions across the line of sight (vertical motions in the case of the rotation curve, radial and azimuthal motions in the case of the vertical studies) can be ignored, and quite modelindependent results can therefore be derived just from radial velocities (and adopted values for our distance to the Galactic center and solar motion). For a discussion of rotation curve measurements, we refer to the article by Merrifield (1993). Here we concentrate on the vertical component of the Galactic force in the solar cylinder.

\subsection{THE VERTICAL GRAVITATIONAL FORCE IN THE SOLAR CYLINDER}

The vertical equilibrium of a stellar population of density $\nu$ and velocity dispersion tensor $\sigma_{i j}^{2}$ in a gravitational field with vertical force $K_{z}$ is described by the Jeans equation:

$$
\frac{1}{\nu} \frac{\partial \nu \sigma_{z z}^{2}}{\partial z}+\frac{1}{\nu} \frac{\partial R \nu \sigma_{R z}^{2}}{\partial R}=K_{z}
$$

The first term is measureable from star counts as a function of distance from the plane and radial velocity measurements towards the Galactic poles. The second term, on the other hand, seems hand-picked to be unobservable. Not only does is require measurements of the space motions of the stars to allow evaluation of the cross-dispersion $\sigma_{R z}^{2}$, but it also involves a radial derivative, which can only be measured by several such measurements in different fields. Fortunately, it is possible to show with simple models that at heights up to $1 \mathrm{kpc}$ this term is unlikely to exceed $10 \%$ of the others (Kuijken and Gilmore, 1989a).

Once the force $K_{z}$ is derived, the corresponding mass density $\rho$ follows from the Poisson equation:

$$
4 \pi G \rho=-\frac{\partial K_{z}}{\partial z}-\frac{1}{R} \frac{\partial R K_{R}}{\partial R} .
$$

Once again, only two terms are important: the final term is exactly zero for a flat rotation curve, and small for plausible rotation curve slopes. 
In practice, the presence of the two derivatives in these equations makes a direct application to real data very unstable. It is preferable to work backwards, by calculating the $K_{z}$-forces corresponding to trial density distributions. These forces can then either be compared to the results of applying the Jeans equation to the smoothed tracer data, or (if the tracer's space density is known with sufficient accuracy) used to predict the kinematics of the tracer as a function of position. Comparison of the predicted kinematics with the observed velocities then allows the best-fitting total density model to be selected (Kuijken and Gilmore, 1989a).

Note that the tracer population kinematics mostly constrain the force $K_{z}$. Since this force is related to the $z$-integral of the total mass density, its measurement mainly constrains the surface mass density of the stars below a particular height, and not the volume mass density at that height. It is therefore much more difficult to measure robustly the total volume density of mass at $z=0, \rho_{0}$, than the surface mass density near the sun. Though results are often reported in terms of values for $\rho_{0}$, such measurements follow from a particular assumed form for the mass distribution, and are rather model-dependent. A popular model is the formulation by Bahcall (1984a) in terms of a factor $P$, the ratio between the mass in dark and identified matter in the disk. He divided the disk stars and gas into a number of isothermal components, all of which respond to each other's gravity, and solved for the self-consistent situation in which each component is in equilibrium with the overall potential generated by all of them together. A number of isothermal dark matter components of various velocity dispersions can also be added in. The " $P$-models" are obtained by multiplying the mass of each disk component by a factor $(1+P)$, i.e. by adding a dark disk of identical kinematics to the observed one. Though these models are very useful parameterizations, it should be borne in mind that they are somewhat restrictive. For instance, Bahcall showed that models preferentially loaded with very low dispersion dark matter (below the dispersion of the tracer population) require much higher values for $\rho_{0}$ than the $P$-models fitted to the same tracer kinematics. Results of $\rho_{0}$ measurements from $K_{z}$ determinations will therefore always be model dependent: it is not possible to constrain the mid-plane density of dark matter populations with dispersion significantly below that of the tracer population. The overall surface density of cold populations is constrained, however.

The choice of tracer population is fundamentally important for this type of study. On the one hand, the stars need to form a steady-state population if they are to serve as a faithful tracer of the potential. Since stars are born in groups, they need to be sufficiently old for this initial correlation to be phase-mixed away. The required timescales for such mixing are surprisingly long, since at low heights the vertical force is almost linear, and phase- 
mixing does not occur in harmonic potentials. For example, the vertical oscillation period of a star with initial velocity of $10 \mathrm{~km} \mathrm{~s}^{-1}$ in the no-darkmatter potential $P=0$ of Bahcall (1984a) is $88 \mathrm{Myr}$, only $10 \%$ longer than the period of a star with infinitesimal initial velocity. Initial spreads in velocity of this amplitude therefore require $\left(80^{-1}-88^{-1}\right)^{-1}=800 \mathrm{Myr}$ to phase-mix. Only stellar populations in which the majority of the stars are older than this limit can therefore be expected to give reliable results. A more practical concern is the requirement to measure accurately the stars' positions above the plane: good distance determinations are essential. Broad-band photometry together with a crude dwarf-giant discrimination works fine for $\mathrm{dK}$ stars, but on the giant branch or near the main sequence turnoff more accurate luminosity measurements, such as uvby $\beta$ or DDO photometry are required.

\begin{tabular}{lcl}
\hline Reference & tracer & $1-\sigma$ result \\
\hline Bahcall, 1984b,c & dF, gK & $P \sim 1$ \\
Bienaymé et al., 1987 & all types & $P=0-0.3$ \\
Kuijken and Gilmore, 1989b,91 (KG) & dK & $\Sigma=48 \pm 9 M_{\odot} \mathrm{pc}^{-2}$ \\
Gould, 1990 & KG dK's & $\Sigma=54 \pm 9 M_{\odot} \mathrm{pc}^{-2}$ \\
Kuijken, 1991 & KG dK's + local & $P=0 \pm 0.3$ \\
Bahcall et al., 1992 (BFG) & gK & $P=0.39-3.42$ \\
Fuchs and Wielen, 1993 & KG dK's + local & $P<0.5$ \\
Flynn and Fuchs, 1994 & BFG gK's + local & $P=0.25 \pm 0.25$ \\
\hline
\end{tabular}

TABLE 1. Measurements of the mass density near the sun over the last decade. $P$ refers to the ratio of dark to identified matter, under the assumption that these are distributed equally; $\Sigma$ is the total inferred surface mass density, [c.f. total identified mass density of $48 \pm 7 M_{\odot} \mathrm{pc}^{-2}$ (Kuijken and Gilmore, 1989a)].

Over the last decade or so, there have been several analyses of the vertical kinematics of various tracer populations. The results of these are summarized in Table 1. Several different analysis techniques, and several different samples of stars, were used in these investigations, but the overall results are in fair agreement: while there may be some dark matter associated with the disk of the galaxy, it probably does not make a serious impact on the total surface density of the disk near the sun.

It is interesting to note that this dynamically inferred surface density is not sufficient to explain the amplitude of the rotation curve at the solar radius. Fig. 1 shows the circular speed at the sun generated by an exponential disk of local surface density $48 \mathrm{M}_{\odot} p c^{-2}$ and a bulge of up to $2 \times 10^{10}$ $\mathrm{M}_{\odot}$ as a function of disk scale length, assuming we are $7.8 \mathrm{kpc}$ from the center of the Galaxy. Unless the Milky Way scale length is below $2.5 \mathrm{kpc}$, 


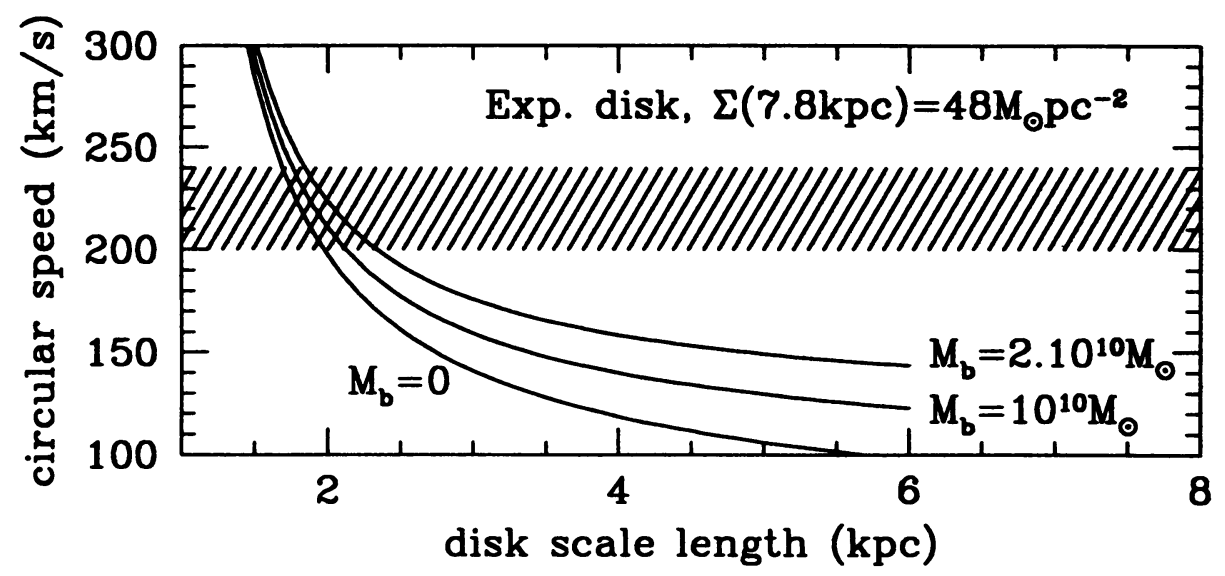

Figure 1. The total circular speed at the sun $\left(R_{0}=7.8 \mathrm{kpc}\right)$ contributed by a disk of local surface density $48 \mathrm{M}_{\odot} p c^{-2}$ and a bulge of 0,1 or $2 \times 10^{10} \mathrm{M}_{\odot}$, as a function of the disk exponential scale length. Plausible values of the circular speed are shaded.

a large part of the radial force in the solar neighbourhood is not accounted for by the disk and bulge. The Milky Way is therefore not close to being a maximal disk galaxy.

\section{Is the Dark Matter a Stellar Population?}

In their original studies of the gravitational potential in the solar neighbourhood, Kapteyn (1922) and Oort (1932) considered their studies as ways of measuring the total mass density in the solar neighbourhood, implicitely assuming that all this mass was stellar. Only later did it become possible to make independent estimates of the mass contained in stars in the Galactic disk, and were the molecular clouds, a major contributor of mass in the Galactic plane, discovered. It is now believed that there is a significant amount of dark matter in the Galaxy, if not as part of the disk population. Can this component consist of stars?

Surveys of the solar neighbourhood have found no evidence of a dramatic increase of the stellar mass function at lower masses. If the physics of star formation and nuclear burning are sufficiently unrelated, it is reasonable to argue that there should not be an abrupt change in the mass function near the hydrogen-burning limit, at a mass near $0.08 \mathrm{M}_{\odot}$. The luminosity function of the faintest main sequence stars appears to be smooth, and extrapolations of the corresponding mass function down to zero mass do not suggest that there is a large mass locked up in such brown dwarf stars (Bessel and Stringfellow, 1993)-furthermore, not a single brown 
dwarf candidate has so far stood the test of time. The luminosity function of white dwarf stars is consistent with a single cooling population, with the oldest white dwarfs having a cooling age of some 6-14 Gyrs (Yuan, 1989; Wood 1992); since the cooling edge of the luminosity function is resolved, older white dwarfs would have to constitute a disjoint population for which there is no direct evidence to date. The identified white dwarf population contributes a significant but not dominant $10 \%$ of the stellar mass in the solar neighbourhood. Moreover, chemical evolution models and the observed type I supernova rate (Wyse, this volume) limit the mass that can at present be locked up in white dwarfs.

The stellar halo of the Milky Way, if it has a mass function which is not strongly biased towards faint, light stars, also does not contribute a dynamically significant amount of matter. Very deep star counts by Richer and Fahlman (1992) and Richstone et al. (1992) constrain the number density of faint metal poor halo stars distributed with a $R^{-2}$ density (which is what is required to generate a flat rotation curve), effectively ruling them out as constituents of the dark halo unless they form a population with mass to light ratio of $\sim 100$. Furthermore, dynamical analyses of globular clusters yield mass to light ratios indicative of a standard mass function with little or no dark matter. A faint stellar halo population responsible for the Milky Way's flat rotation curve would therefore have to be distinct from the globular cluster population.

There are therefore no direct indications that the Milky Way contains a dark stellar population.

\subsection{MICROLENSING SURVEYS}

In the last few years, the first results from the microlensing surveys have appeared (Alcock et al., 1993; Aubourg et al., 1993; Udalski et al., 1994). These surveys look for the temporary brightening that takes place when a background star's light is gravitationally lensed by an intervening object, an effect first noted to be observable by Paczyński (1986). The current surveys are sensitive to dark (and luminous) objects in the (sub-)stellar mass range. Fields in two directions have been observed in great detail: one set in the Magellanic Clouds, and one set towards the Galactic bulge. If the number of events has been disappointingly low towards the Clouds, it has surpassed all expectations in the direction towards the Bulge.

So far, it is mainly the total "optical depth" which is reasonably welldefined by the data. This is simply the total fraction of time that any one star is amplified by at least $0.34^{\mathrm{m}}$. Such amplification occurs whenever a foreground "lens" enters a slender cigar-shaped volume called the "microlensing tube", which surrounds the line of sight and has radius (the 
"Einstein radius")

$$
R_{E}(x)=2 \sqrt{G m x(1-x / L)} / c \propto \sqrt{m},
$$

where $x$ and $L$ are the distance of the observer to the intervening lens and to the background star, respectively. The optical depth due to a population of objects of mass $m$ with number density $\nu$ is then

$$
\tau=\int_{0}^{L} d x \pi R_{E}^{2} \nu(x) \propto m^{0} .
$$

Thus the total optical depth is independent of the mass of the lensing objects (for a given mass density, $\nu \propto m^{-1}$ ), though the detection efficiency is a strong function of $m$ via the event duration. Currently the uncertaincies in detection efficiency limit the accuracy of the measurement of $\tau$. The event rate $\Gamma$ is the rate at which lenses enter the microlensing tube:

$$
\Gamma=\int_{0}^{L} d x 2 R_{E}(x) \nu(x) v_{\perp} \propto v_{\perp} / \sqrt{m} .
$$

Notice that it is only the event rate (equivalently, the typical event duration, given an observed total optical depth) which can constrain the mass of the microlenses: statistical uncertainties aside, masses determined from the event rate will also involve a factor $v_{\perp}^{2}$ and so depend on the kinematics assumed for the dark matter. (There are ways of measuring parallaxes and proper motions from individual events, involving more sophisticated observations; Gould, 1994).

The observed optical depth towards the LMC is about a factor 10 smaller than it would be if (1) the halo were round and had a flat rotation curve out to the LMC, and (2) the dark matter were in objects of the right mass to produce detectable microlensing. The tentative conclusion is therefore that the dark halo of the Galaxy either is flattened, or consists of very low-mass objects (perhaps elementary particles) which do not produce significant lensing.

The high event rate towards the Galactic bulge is a surprise. If the mass corresponding to the observed optical depth of $3 \times 10^{-6}$ (Udalski et $a l ., 1994)$ is distributed uniformly along the line of sight out to a distance of $8 \mathrm{kpc}$, it must have a total mass density of $0.5 \mathrm{M}_{\odot} p c^{-3}$. Such high mass densities close to the plane are not only in conflict with the measurements of the dark matter fraction in the solar neighbourhood, but on their own already imply too high a rotation velocity for the Galaxy. While these results are at present still a bit of a mystery, it seems possible that more careful modelling in which the effects of lensing of far-side bulge stars by near-side ones is properly taken into account, can increase the expected 


\begin{tabular}{r|rrrrr} 
& \multicolumn{5}{|c}{ BAR AXIS RATIO } \\
$\alpha$ & 1.0 & 1.5 & 2.0 & 2.5 & 3.0 \\
\hline-1 & 0.79 & 1.14 & 1.41 & 1.61 & 1.76 \\
-2 & 0.65 & 0.86 & 1.00 & 1.08 & 1.14 \\
-3 & 0.52 & 0.61 & 0.64 & 0.64 & 0.62 \\
-4 & 0.40 & 0.40 & 0.36 & 0.32 & 0.29 \\
\hline
\end{tabular}

TABLE 2. Total optical depths in units of $10^{-6}$ towards Baade's window due to self-lensing by the bulge/bar only, assuming that the bulge is a factor $q$ more extended along the line of sight than it appears in projection. The luminosity function of the background stars is assumed to be a power law of index $\alpha$.

optical depth considerably (Kiraga and Paczyński, 1994). Further modelling freedom is provided by the evidence for a stellar bar in the central kpc, the orientation of which may enhance bulge-bulge lensing greatly (stretching the bulge down the line of sight places the near side of the bulge in a much fatter part of the microlensing tube for sources on the far side). Table 2 shows the optical depths from bulge star lensing by other bulge stars, derived by stretching Kent's (1992) model a factor $q$ along the line of sight. Unfortunately the bar complicates the kinematics in the central regions of the Milky Way, making it harder to translate the observed event durations into lens masses. It does seem, though, that the observed rates are plausibly explained by objects in the conventional stellar mass range.

All in all, it appears likely that the microlensing surveys will end up telling us a lot about the stellar makeup of the inner Galaxy.

\section{Summary}

This review has concentrated on two questions, "How can stellar populations be used to constrain the dark matter distribution?" and "Is the dark matter in the Milky Way a stellar population?". The first question can be answered with reference to a long history of investigations of stellar kinematics of many different types, current results of which appear to be converging on the view that while there is certainly a large amount of dark matter in the Milky Way, little or none of it forms part of the Galactic disk. The second question is one that is nowadays being addressed afresh, with microlensing surveys towards the LMC and the bulge providing fresh information on the mass distribution of the Galaxy. Current results favour models in which the halo does not consist of stellar mass objects. The high rates observed towards the bulge may be a result of significant lensing by 
near-side bulge stars, especially if the inner Galaxy contains a stellar bar.

\section{References}

Alcock, C. et al., 1993, Nature 365, 621

Aubourg, E. et al., 1993, Nature 365, 623

Bahcall, J.N., 1984a, Ap. J. 276, 156

Bahcall, J.N., 1984b, Ap. J. 276, 169

Bahcall, J.N., 1984c, Ap. J. 287, 926

Bahcall, J.N., Flynn, C. and Gould, A., 1992, Ap. J. 389, 234

Bessel, M.S. and Stringfellow, G.S., 1993, Ann. Rev. Astron. Astroph. 31, 433

Bienaymé, O., Robin, A.C. and Crézé, 1987, A. B A. 180, 94

Comeron, F., Torra, J., Gomez, A. E., 1992, Astroph \& Spa. Sci. 187, 187

Fich, M. and Tremaine, S., 1991, Ann. Rev. Astron. Astroph. 29, 409

Flynn, C. and Fuchs, B., 1994, M. N. R. A. S., in press

Fuchs, B. and Wielen, R., 1993, Back to the Galaxy, eds. S.S. Holt and F. Verter, p. 580 Gould, A., 1990, M. N.R. A. S. 244, 25

Gould, A., 1994, preprint

Hartkopf, W.I. and Yoss, K.M., 1982, A. J. 87, 1679

Hill, G., Hilditch, R.W. and Barnes, J.V., 1979, M. N. R. A. S. 186, 813

Kapteyn, J.C., 1922, Ap. J. 55, 302

Kent, S.M., 1992, Ap. J. 387, 181

Kiraga, M. and Paczyński, B., 1994, Ap. J. 430, L101

Kuijken, K., 1991, Ap. J. 372, 125

Kuijken, K. and Gilmore, G., 1989a, M. N. R. A. S. 239, 605

Kuijken, K. and Gilmore, G., 1989b, M. N. R. A. S. 239, 650

Kuijken, K. and Gilmore, G., 1991, Ap. J. 367, L9

Merrifield, M.R., 1993, Back to the Galaxy, eds. S.S. Holt and F. Verter, p.437

Oort, J.H., 1932, Bull. Astron. Neth. 6, 249

Paczyński, B., 1986, Ap. J. 304, 1

Richstone, D., Gould, A., Guhathakurta, P. and Flynn, C., 1992, Ap. J. 388, 354

Richer, H.B. and Fahlman, G.G., 1992, Nature 358, 383

Schechter, P. 1993, Back to the Galaxy, eds. S.S. Holt and F. Verter, p. 571

Udalski, A., et al., 1994, Acta Astronomica 44, 165.

Wood, M.A., 1992, Ap. J. 386, 539

Yuan, J.W., 1989, A. \& A. 224, 108

SELLWOOD: You wanted to increase the lensing rate in the OGLE survey by making the bulge elongated along the line of sight. What range of angles and axis ratios are required for the bar to push up the event rate to that observed?

KUIJKEN: The important parameters are the length of the bulge along our line of sight compared to the projected size (on which the axisymmetric models are based). The rate can therefore only be increased as long as the long axis of the bulge points within $45^{\circ}$ of the sun. The precise value of the enhancement factor over the self-lensing rate from an axisymmetric bulge depends on the luminosity function and detection limits of the lensing events, but it can reach a factor of two.

RENZINI: You have assumed a flat distribution of disk stars towards Baade's window. In a recent paper, the OGLE collaboration has shown that the disk stars closely concentrate within the $2 \mathrm{kpc}$ spiral arm, with very few disk stars beyond it. They also show that the B-S model gives a very poor fit to the actual star counts in Baade's Window. 
KUIJKEN: These findings are all the more reason for considering the self-lensing rate by bulge/bar stars. 